\title{
On exchangeable continuous variable systems
}

\author{
Robert König $^{1, a)}$ and Michael M. Wolf ${ }^{2,3}$ \\ ${ }^{1}$ Institute for Quantum Information, Caltech, Pasadena, CA 91125, USA \\ ${ }^{2}$ Max-Planck-Institut für Quantenoptik, D-85748 Garching, Germany \\ ${ }^{3}$ Niels Bohr Institute, DK-2100 Copenhagen, Denmark
}

(Received 17 September 2008; accepted 18 November 2008; published online 8 January 2009)

\begin{abstract}
We investigate permutation-invariant continuous variable quantum states and their covariance matrices. We provide a complete characterization of the latter with respect to permutation invariance and exchangeability and representing convex combinations of tensor power states. On the level of the respective density operators this leads to necessary criteria for all these properties which become necessary and sufficient for Gaussian states. For these we use the derived results to provide de Finetti-type theorems for various distance measures. (C) 2009 American Institute of Physics. [DOI: 10.1063/1.3043788]
\end{abstract}

\section{INTRODUCTION}

A density operator on an $n$-partite system $\left(\mathrm{C}^{d}\right)^{\otimes n}$ is called permutation-invariant or symmetric if it is invariant under interchanges of the subsystems, i.e., conjugation by permutations. A $k$-partite density operator on $\left(C^{d}\right)^{\otimes k}$ is called n-exchangeable if it is the partial trace of a permutation-invariant state on $\left(\mathrm{C}^{d}\right)^{\otimes n}$. According to the quantum de Finetti theorem, such states can be approximated by convex combinations of $k$-fold product states with error of order $d^{2} k / n$. This result has various applications in quantum information theory (e.g., in security proofs for cryptographic protocols ${ }^{1,2}$ or the justification of mean-field approaches ${ }^{3}$ ) and relatives [the "monogamy" of entanglement (see Refs. 4 and 5) or the appearance of a local classical description under symmetries $\left.{ }^{6-8}\right]$.

Unfortunately, such de Finetti-type statements for finitely exchangeable states are no longer true when the Hilbert space $\mathrm{C}^{d}$ of an individual ("local") system is replaced by an infinitedimensional Hilbert space-as it happens, for instance, when dealing with fields of light. Indeed, Corollary III.9 in Ref. 9 provides examples of $n$-exchangeable states on $\left(C^{d}\right)^{\otimes k}$ whose distance from any convex combination of product states is of order at least $d / n$. This rules out the possibility of there being a de Finetti theorem for all finitely exchangeable states on infinitedimensional Hilbert spaces.

It is, however, still possible to find de Finetti-type results for infinite-dimensional systems by considering restricted classes of states or observables. (See Refs. 10 and 11 for a different direction of generalization of the basic de Finetti claim for quantum states.) An example are the coherent cat-states for which a de Finetti theorem was obtained by D'Cruz et al. ${ }^{12}$ an "exponential" version thereof is provided in Ref. 13. In the present work we will first restrict the set of observables and consider covariance matrices of canonical field operators (such as position and momentum or the quadratures of a field of light). In the second part we will then use these results in order to derive de Finetti-type theorems for a restricted class of states, namely, Gaussian states. These play an important role in quantum optics because coherent, squeezed, and their thermal states are all Gaussian and remain so under the action of quadratic Hamiltonians and homodyne measurements.

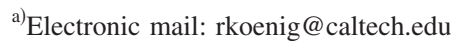


Symmetry and exchangeability for infinite-dimensional systems are defined analogously to the finite-dimensional case: We substitute the local Hilbert spaces $\mathbb{C}^{d}$ by the Hilbert space $\mathcal{H}_{d}$ $:=\mathcal{H}^{\otimes d}$ of $d$ harmonic oscillators or modes with $\mathcal{H} \cong \mathcal{L}^{2}$ being infinite dimensional. The symmetric group $\mathrm{S}_{n}$ acts on the $n$-fold tensor product $\mathcal{H}_{d}^{\otimes n}$ by permuting the factors. Pure states invariant under this action are commonly referred to as Bose-symmetric states. Here we focus on a larger class of states that results when considering the action of $\mathrm{S}_{n}$ on the set $\mathcal{B}\left(\mathcal{H}_{d}^{\otimes n}\right)$ of bounded operators on $\mathcal{H}_{d}^{\otimes n}$ by conjugation: A density operator $\rho$ on $\mathcal{H}_{d}^{\otimes n}$ is called symmetric if it is invariant under this action, i.e., if $\pi \rho \pi^{\dagger}=\rho$ for all $\pi \in \mathrm{S}_{n}$. States $\operatorname{tr}_{\mathcal{H}_{d}^{\otimes n-k} \rho} \rho$ on $\mathcal{H}_{d}^{\otimes k}$ obtained by tracing out $n-k$ local systems of a symmetric state $\rho$ on $\mathcal{H}_{d}^{\otimes n}$ are called n-exchangeable. Infinitely exchangeable states on $\mathcal{H}_{d}^{\otimes k}$ are those that are $n$ exchangeable for any $n \geq k$; according to the infiniteexchangeability de Finetti theorem, ${ }^{14-16}$ which also holds on infinite-dimensional Hilbert spaces, these are all states of the form $\int \tau^{\otimes k} d m(\tau)$, i.e., convex combinations of $k$-fold product states $\tau^{\otimes k}$, where $\tau$ are states on the single system $\mathcal{H}_{d}$. We call such convex combinations power states.

We will provide some prerequisites in the next section and then give a detailed discussion of permutation-invariant covariance matrices. This will yield necessary criteria for the respective density matrices which become sufficient as well when considering Gaussian states. de Finetti theorems for the latter are then provided in the second part.

\section{PRELIMINARIES}

This section will introduce the notation and recall basic results (see Ref. 17) on covariance matrices and the corresponding continuous variable states. Consider a system of $n$ sites, each of which with $d$ canonical degrees of freedom (modes). Such a system can be described using a symplectic vector space, the phase space, $\mathcal{V} \cong \mathbb{R}^{2 d} \otimes \mathbb{R}^{n}$ of dimension $2 d \cdot n$. We choose canonical coordinates such that the symplectic matrix $\sigma$ has the form

$$
\sigma=\left(\begin{array}{cc}
0_{d} & -1_{d} \\
1_{d} & 0_{d}
\end{array}\right) \otimes 1_{n}
$$

where $0_{d}$ and $\mathbb{1}_{d}$ are the $d \times d$ zero and identity matrices, respectively. Occasionally, we will denote with a slight abuse of notation the symplectic matrix for a subsystem (less than $n$ sites) by $\sigma$ as well. The block structure in Eq. (1) reflects the grouping into position and momentum coordinates. The corresponding quantum system with Hilbert space $\mathcal{H}_{d}^{\otimes n} \cong\left(\mathcal{L}^{2}\right)^{\otimes d n}$ is characterized by canonical position and momentum operators $\left\{R_{i}^{\alpha}\right\}_{\alpha=1}^{2 d}$ for each site $i=1, \ldots, n$; here $\left\{R_{i}^{\alpha}, R_{i}^{d+\alpha}\right\}$ for $1 \leq \alpha \leq d$ are the position and momentum operators of the $\alpha$ th mode of the $i$ th site acting nontrivially only on the $i$ th factor of the $n$-fold tensor product space $\mathcal{H}_{d}^{\otimes n}$. These operators satisfy the canonical commutation relations (CCRs)

$$
i\left[R_{k}^{\alpha}, R_{l}^{\beta}\right]=\sigma_{(\alpha, k),(\beta, l)} 1 .
$$

Real linear transformations $S: \mathcal{V} \rightarrow \mathcal{V}$ which preserve the CCR (i.e., $S \sigma S^{T}=\sigma$ ) are called symplectic. Affine transformations are obtained from the unitary Weyl operators $W(\xi):=e^{i \xi^{T} \sigma R}$. (Here we assume, as usual, the Weyl system to be irreducible and strongly continuous.) These give rise to displacements

$$
\left.W(\xi) R_{i}^{\alpha} W(\xi)^{\dagger}=R_{i}^{\alpha}+\xi_{i}^{\alpha}\right\rfloor .
$$

These only alter the first moments of a state, collected in the displacement vector $D:=\operatorname{tr}(\rho R)$, while keeping untouched the covariance matrix $(\mathrm{CM}) \Gamma$ with entries

$$
\Gamma_{(\alpha, i),(\beta, j)}=\operatorname{tr}\left(\left\{R_{i}^{\alpha}-D_{i}^{\alpha} \cdot 1, R_{j}^{\beta}-D_{j}^{\beta} \cdot 1\right\}_{+} \rho\right) .
$$

Since the Weyl operators act locally in the sense that they factorize with respect to local systems we can without loss of generality typically assume $D=0$. Positivity of the density matrices 
implies that any $\mathrm{CM}$ has to satisfy $\Gamma \geq i \sigma$. Conversely, every real symmetric matrix fulfilling this requirement is a valid CM. In particular, there always exists a Gaussian state which is, up to displacements, completely characterized by this CM.

\section{CHARACTERIZATION OF COVARIANCE MATRICES}

We are now ready to characterize covariance matrices with respect to permutation invariance, exchangeability, and power-state property. Clearly, we could express all results below in terms of Gaussian states. However, we want to logically separate the treatment of CMs since it is neither necessary for the state to be Gaussian nor does the corresponding density matrix have to share the full symmetry of the CM.

We will say that a $\mathrm{CM}$ is a power-state $\mathrm{CM}$ if it corresponds to a mixture of states with product CMs of the form $\gamma \otimes 1_{n}$. Similarly, we say that it is $n$-exchangeable if it is a sub-block of a permutation-invariant $\mathrm{CM}$ of $n$ sites, and we call it separable if it corresponds to a mixture of states with product $\mathrm{CMs}$ of the form $\oplus_{i=1}^{n} \gamma_{i}$.

The main ingredient of our analysis is the following one-to-one correspondence between permutation-invariant CMs and bipartite product CMs.

Proposition 1 (Symmetric CMs): Let $X$ be any real orthogonal $n \times n$ matrix for which $X_{i, 1}$ $=1 / \sqrt{n}$ for all $i=1, \ldots, n$. Then $S=1_{2 d} \otimes X$ is a symplectic transformation on $\mathcal{V}=\mathbb{R}^{2 d} \otimes \mathbb{R}^{n}$ which gives rise to a one-to-one mapping between permutation-invariant $\mathrm{CMs} \Gamma$ on $\mathcal{V}$ and pairs of CMs $\mathbf{E}, \mathbf{F}$ on $\mathbb{R}^{2 d}$ such that

$$
\Gamma=\Gamma(\mathbf{E}, \mathbf{F})=S\left(\mathbf{E} \oplus \bigoplus_{i=2}^{n} \mathbf{F}\right) S^{T} .
$$

Proof: Evidently, $S$ is a symplectic transformation and therefore maps CMs onto CMs. In fact, it is a passive transformation, i.e., number preserving. We first show that every pair of CMs E, F leads to a permutation-invariant $\Gamma$. For this we have to show that the entries of the latter are site independent in the sense that

$$
\Gamma_{(\alpha, i),(\beta, i)}=A_{\alpha, \beta}, \quad \Gamma_{(\alpha, i),(\beta, j)}=B_{\alpha, \beta} \quad \text { for } i \neq j .
$$

The symmetric $2 d \times 2 d$ matrices $A$ and $B$ encode the local (on-site) and nonlocal (intersite) correlations, respectively. With some abuse of Dirac notation, Eq. (4) can be written as

$$
\Gamma=E \otimes X|1\rangle\langle 1| X^{T}+F \otimes\left(1-X|1\rangle\langle 1| X^{T}\right) .
$$

Exploiting the condition on the first column of $X$, this leads to

$$
\Gamma_{(\alpha, i),(\beta, j)}=\frac{1}{n} \mathbf{E}_{\alpha, \beta}+\left(\delta_{i, j}-\frac{1}{n}\right) \mathbf{F}_{\alpha, \beta},
$$

which is indeed permutation-invariant. This immediately implies that every permutation-invariant CM characterized by $A, B$ can be obtained in the above way by choosing

$$
\mathbf{E}=A+(n-1) B \text { and } \mathbf{F}=A-B .
$$
CMs.

We will now use this representation in order to characterize power-state and exchangeable

Proposition 2 (Power-state CMs): Let $\Gamma(\mathbf{E}, \mathbf{F})$ be a permutation-invariant $\mathrm{CM}$ on $\mathbb{R}^{2 d} \otimes \mathbb{R}^{n}$. Then the following are equivalent: (i) $\Gamma$ is a power-state $\mathrm{CM}$, (ii) $\Gamma$ is a separable $\mathrm{CM}$, and (iii) there exists a $\mathrm{CM} \gamma$ of $d$ modes such that $\mathbf{E} \geq \gamma$ and $\mathbf{F} \geq \gamma$.

Proof: Clearly, (i) implies (ii). The remaining proof closely follows the derivation of the separability criterion for Gaussian states in Ref. 18 . There it is shown that for every separable CM $\Gamma$ there exist CMs $\gamma_{i}$ such that $\Gamma \geq \oplus_{i=1}^{n} \gamma_{i}$. Averaging over all permutations, we obtain $\Gamma \geq \gamma \otimes \mathbb{1}_{n}$ with $\gamma=(1 / n) \Sigma_{i} \gamma_{i}$. Applying the symplectic transformation of Proposition 1 to this 
inequality leads to $\mathbf{E}, \mathbf{F} \geq \gamma$ (since the right hand side has $\mathbf{E}=\mathbf{F}=\gamma$ ) and thus (iii) is equivalent to (ii). Finally, again following Ref. $18 \Gamma \geq \gamma \otimes 1_{n}$ implies that $\Gamma$ is the $\mathrm{CM}$ of a mixture of states with $\mathrm{CM} \gamma \otimes 1_{n}$.

Proposition 3 (Exchangeable CMs): Let $\Gamma$ be a permutation-invariant $\mathrm{CM}$ on $\mathbb{R}^{2 d} \otimes \mathbb{R}^{k}$, $k \leq n$. With the notation from Proposition $1 \Gamma$ is $n$-exchangeable if and only if $A+(n-1) B \geq i \sigma$ or equivalently $\mathbf{E}=(k / n) \widetilde{\mathbf{E}}+(1-k / n) \mathbf{F}$ with $\widetilde{\mathbf{E}}$ being any $\mathrm{CM}$ of $d$ modes.

This follows in a straightforward way from Proposition 1 and the relation Eq. (8). It is remarkable that Proposition 3 gives a simple criterion for deciding $n$-exchangeability for any CM. Note that such a criterion is not known for general exchangeable states on finite-dimensional Hilbert spaces (see Ref. 19).

We also point out that according to Proposition $3, \Gamma$ admits a permutation-invariant supersystem of $n$ sites for all $n \geq k$ if and only if $\mathbf{E} \geq \mathbf{F}$; this is a stronger condition than the one for power-state CMs.

\section{IV. de FINETTI THEOREMS FOR GAUSSIAN STATES}

We now step back from phase space to Hilbert space and derive upper bounds on the distance between exchangeable Gaussian states and the set of power states. Note that the conditions for a Gaussian state to be exchangeable or a power-state are precisely those given in Sec. III. We begin with the single-mode case $(d=1)$ and recall that the trace norm $\left(L^{1}\right.$ norm) is defined by $\|A\|_{1}$ $=\operatorname{tr} \sqrt{A^{\dagger} A}$.

Theorem 1 (Single-mode de Finetti): Let $\rho_{\Gamma(\mathbf{E}, \mathbf{F})}$ be an $n$-exchangeable Gaussian density matrix describing a permutation-invariant $k \leq n$ mode system. Define $\mathbf{E}^{\prime}=[n /(n-k)] \mathbf{E}$. Then $\rho_{\Gamma\left(\mathbf{E}^{\prime}, \mathbf{F}\right)}$ is a Gaussian power state, and

$$
\left\|\rho_{\Gamma(\mathbf{E}, \mathbf{F})}-\rho_{\Gamma\left(\mathbf{E}^{\prime}, \mathbf{F}\right)}\right\|_{1} \leq\left(\frac{n}{k}-\frac{1}{2}\right)^{-1} .
$$

Proof: The matrix $\mathbf{E}^{\prime}$ defines a valid CM, since multiplying by a factor $\lambda:=n /(n-k) \geq 1$ preserves the condition $\mathbf{E} \geq i \sigma$. Using $n$-exchangeability and the $C M \mathbb{E}$ appearing in Proposition 3 we can write $\mathbf{E}^{\prime}=[k /(n-k)] \widetilde{\mathbf{E}}+\mathbf{F}$. Observe that $\widetilde{\mathbf{E}}$ is a positive semidefinite operator, which is implied by $\tilde{\mathbf{E}} \geq i \sigma$ (via complex conjugation and averaging). We conclude that $\mathbf{E}^{\prime} \geq \mathbf{F}$. According to Proposition 2, this implies that $\rho_{\Gamma\left(\mathbf{E}^{\prime}, \mathbf{F}\right)}$ is indeed a Gaussian power-state.

To compute the distance between $\rho_{\Gamma\left(\mathbf{E}^{\prime}, \mathbf{F}\right)}$ and $\rho_{\Gamma(\mathbf{E}, \mathbf{F})}$, we use the tensor product forms $\rho_{\Gamma\left(\mathbf{E}^{\prime}, \mathbf{F}\right)}=\rho_{\mathbf{E}^{\prime}} \otimes \rho_{\mathbf{F}}^{\otimes k-1}$ and $\rho_{\Gamma(\mathbf{E}, \mathbf{F})}=\rho_{\mathbf{E}} \otimes \rho_{\mathbf{F}}^{\otimes k-1}$ of the states in the basis of Proposition 1 together with the stability property $\left\|\rho \otimes \sigma-\rho^{\prime} \otimes \sigma\right\|_{1}=\left\|\rho-\rho^{\prime}\right\|_{1}$ of the trace norm, so that

$$
\left\|\rho_{\Gamma(\mathbf{E}, \mathbf{F})}-\rho_{\Gamma\left(\mathbf{E}^{\prime}, \mathbf{F}\right)}\right\|_{1}=\left\|\rho_{\mathbf{E}}-\rho_{\mathbf{E}^{\prime}}\right\|_{1} .
$$

To evaluate this further we use that there is a canonical basis where $\mathbf{E}=s \rrbracket_{2}$ for some $s \geq 1$ and thus $\left.\mathbf{E}^{\prime}=\lambda s\right\rfloor_{2}$. The corresponding density matrices are now simultaneously diagonal in Fock state basis (labeled by $\ell=0, \ldots, \infty)$ with eigenvalues $\mu_{\ell}(s):=2(s-1)^{\ell} /(s+1)^{\ell+1}$ and $\mu_{\ell}(\lambda s)$, respectively. Hence, we proceed with

$$
\left\|\rho_{\mathrm{E}}-\rho_{\mathbf{E}^{\prime}}\right\|_{1}=\sum_{\ell=0}^{\infty}\left|\mu_{\ell}(s)-\mu_{\ell}(\lambda s)\right|=\max _{\ell \in \mathbb{N}} 2\left[\left(\frac{\lambda s-1}{\lambda s+1}\right)^{\ell+1}-\left(\frac{s-1}{s+1}\right)^{\ell+1}\right] .
$$

Equation (10) is evaluated by first noting that $\mu_{\ell}(s)$ and $\mu_{\ell}(\lambda s)$ are normalized geometric series such that one is larger than the other up to some specific $\ell$. In this way the absolute value can be expressed as a difference of two geometric series.

In order to obtain a bound which is independent of $s$ we consider the worst case, i.e., we take the supremum over $s \geq 1$. For this we need to evaluate the expression at the optimal $\ell$ which is one of the two integers next to 


$$
\left(\log \left(\frac{\log \nu(s)}{\log \nu(\lambda s)}\right) / \log \left(\frac{\nu(\lambda s)}{\nu(s)}\right)\right)-1,
$$

with $\nu(s):=(s-1) /(s+1)$. The supremum of (10) is achieved for $s=1$ for which $\ell=0$ attains the maximum so that finally

$$
\left\|\rho_{\mathrm{E}}-\rho_{\mathrm{E}}\right\|_{1} \leq 2 \frac{\lambda-1}{\lambda+1}=\left(\frac{n}{k}-\frac{1}{2}\right)^{-1}
$$

Let us now consider Gaussian systems with several modes per site $(d \geq 1)$. In principle we could follow the same route as above. However, computing the trace norm distance directly appears to become cumbersome due to its nonadditivity with respect to tensor products. For this reason we choose two different figures of merit for measuring the distance to the set of powerstates: the relative entropy ${ }^{20} S\left(\rho, \rho^{\prime}\right)=\operatorname{tr}\left[\rho\left(\log \rho-\log \rho^{\prime}\right)\right]$ and the fidelity $F\left(\rho, \rho^{\prime}\right)=\operatorname{tr} \sqrt{\sqrt{\rho} \rho^{\prime}} \sqrt{\rho}$. Both have benign behavior under taking tensor products (they are additive and multiplicative, respectively) and both yield bounds for the trace norm distance since

$$
\frac{1}{2}\left\|\rho-\rho^{\prime}\right\|_{1}^{2} \leq\left\{\begin{array}{c}
S\left(\rho, \rho^{\prime}\right), \\
2-2 F\left(\rho, \rho^{\prime}\right)^{2} .
\end{array}\right.
$$

Theorem 2 (Multimode de Finetti): Let $\rho_{\Gamma(\mathbf{E}, \mathbf{F})}$ be an $n$-exchangeable Gaussian density matrix describing a permutation-invariant system of $k \leq n$ sites of $d$ modes each. Then the Gaussian power-state $\rho_{\Gamma\left(\mathbf{E}^{\prime}, \mathbf{F}\right)}$ with $\mathbf{E}^{\prime}=[n /(n-k)] \mathbf{E}$ satisfies

$$
\begin{gathered}
S\left(\rho_{\Gamma(\mathbf{E}, \mathbf{F})}, \rho_{\Gamma\left(\mathbf{E}^{\prime}, \mathbf{F}\right)}\right) \leq \log \left(\frac{n-k / 2}{n-k}\right)^{d}, \\
F\left(\rho_{\Gamma(\mathbf{E}, \mathbf{F})}, \rho_{\Gamma\left(\mathbf{E}^{\prime}, \mathbf{F}\right)}\right) \geq\left(\frac{n-k}{n-k / 2}\right)^{d / 2} .
\end{gathered}
$$

Proof: It is clear from the arguments in Theorem 1 that $\Gamma\left(\mathbf{E}^{\prime}, \mathbf{F}\right)$ indeed corresponds to a Gaussian power-state. We again exploit that the distance between $\rho_{\Gamma(\mathbf{E}, \mathbf{F})}$ and $\rho_{\Gamma\left(\mathbf{E}^{\prime}, \mathbf{F}\right)}$ equals that between $\rho_{\mathrm{E}}$ and $\rho_{\mathrm{E}^{\prime}}$. Moreover, we can simultaneously diagonalize the latter two states such that $\rho_{\mathrm{E}}=\otimes_{\alpha=1}^{d} \rho\left(s_{\alpha}\right)$ where each $\rho\left(s_{\alpha}\right)=\sum_{\ell=0}^{\infty} \mu_{\ell}\left(s_{\alpha}\right)|\ell\rangle\langle\ell|$ is a thermal Gaussian state with CM $s_{\alpha} 1_{2}$ and the $s_{\alpha} \geq 1$ are the symplectic eigenvalues of $\mathbf{E}$. For $\mathbf{E}^{\prime}$ we only have to replace $s_{\alpha}$ by $\lambda s_{\alpha}$ with $\lambda=n /(n-k)$. For the relative entropy, summing up the series leads to

$$
\begin{aligned}
S\left(\rho_{\Gamma(\mathbf{E}, \mathbf{F})}, \rho_{\Gamma\left(\mathbf{E}^{\prime}, \mathbf{F}\right)}\right)=\sum_{\alpha=1}^{d} S\left(\rho\left(s_{\alpha}\right), \rho\left(\lambda s_{\alpha}\right)\right)= & \frac{1}{2} \sum_{\alpha=1}^{d}\left(\left(s_{\alpha}-1\right) \log \left(s_{\alpha}-1\right)-\left(s_{\alpha}+1\right) \log \left(s_{\alpha}+1\right)\right. \\
& \left.+\left(s_{\alpha}+1\right) \log \left(\lambda s_{\alpha}+1\right)-\left(s_{\alpha}-1\right) \log \left(\lambda s_{\alpha}-1\right)\right) .
\end{aligned}
$$

For every $\lambda \geq 1$ each summand is a decreasing function in $s_{\alpha}$ such that the supremum is again obtained for $s_{\alpha}=1$, i.e.,

$$
\sup _{s \geq 1} S(\rho(s), \rho(\lambda s))=\log \frac{1+\lambda}{2},
$$

which concludes the proof for the relative entropy upon inserting $\lambda$. In a similar vein we can evaluate the fidelity

$$
F\left(\rho_{\Gamma(\mathbf{E}, \mathbf{F})}, \rho_{\Gamma\left(\mathbf{E}^{\prime}, \mathbf{F}\right)}\right)=\prod_{\alpha=1}^{d} F\left(\rho\left(s_{\alpha}\right), \rho\left(\lambda s_{\alpha}\right)\right),
$$




$$
F(\rho(s), \rho(\lambda s))=\sum_{\ell=0}^{\infty} \sqrt{\mu_{\ell}(s) \mu_{\ell}(\lambda s)}=\frac{2}{\sqrt{(s+1)(\lambda s+1)}-\sqrt{(s-1)(\lambda s-1)}} .
$$

Again the worst case, now the infimum over $s \geq 1$, is attained at $s=1$ which leads to the desired result.

\section{CONCLUSIONS AND OPEN PROBLEMS}

The bounds given in Theorems 1 and 2 are tight for the given class of ansatz states and the proofs allow for any explicitly given CM E to compute the exact distance to this class. Whether the chosen class is optimal or yields at least an optimal scaling remains open. Similarly, the question for which figure of merit a power-state closest to a given $n$-exchangeable Gaussian state can again be chosen to be Gaussian remains open as standard arguments, e.g., based on the central limit theorem (CLT), ${ }^{21}$ do not immediately apply. Some kind of marriage between the CLT and the de Finetti theorem would also be desirable in order to extend the latter in a reasonable way beyond the class of Gaussian states.

Another interesting direction of future research could be to use more general approximating states than Gaussian power-states in the de Finetti theorem. In this way it might be possible to obtain an exponentially small error as in the "almost-product" de Finetti theorem for finitedimensional systems ${ }^{1}$ (see also Refs. 13 and 22). This would be important for applications to continuous variable quantum key distribution, for example. ${ }^{23}$

\section{ACKNOWLEDGMENTS}

R.K. would like to thank the Max Planck Institute for its hospitality and Ignacio Cirac and Geza Giedke for helpful discussions. He acknowledges support by NSF under Contract No. PHY0456720 and PHY-0803371. M.M.W. acknowledges financial support by QUANTOP and the Danish Natural Science Research Council (FNU). Both authors thank the editors and reviewers for their comments.

\footnotetext{
${ }^{1}$ R. Renner, Ph.D. thesis, ETH Zurich, 2005, e-print arXiv:quant-ph/0512258.

${ }^{2}$ K. Horodecki, M. Horodecki, P. Horodecki, D. Leung, and J. Oppenheim, Phys. Rev. Lett. 100, 110502 (2008), e-print arXiv:quant-ph/0712.2265.

${ }^{3}$ G. A. Raggio and R. F. Werner, Helv. Phys. Acta 62, 980 (1989).

${ }^{4}$ T. Osborne and F. Verstraete, Phys. Rev. Lett. 96, 220503 (2006).

${ }^{5}$ M. Wolf, F. Verstraete, and J. Cirac, Phys. Rev. Lett. 92, 087903 (2004).

${ }^{6}$ R. Werner, Lett. Math. Phys. 17, 359 (1989).

${ }^{7}$ M. Wolf, F. Verstraete, and J. Cirac, Int. J. Quantum Inf. 1, 465 (2003).

${ }^{8}$ B. Terhal, A. Doherty, and D. Schwab, Phys. Rev. Lett. 90, 157903 (2003).

${ }^{9}$ M. Christandl, R. König, G. Mitchison, and R. Renner, Commun. Math. Phys. 273, 473 (2007).

${ }^{10} \mathrm{M}$. Christandl and B. Toner, "Finite de finetti theorem for conditional probability distributions describing physical theories," e-print arXiv:quant-ph/0712.0916.

${ }^{11}$ J. Barrett and M. Leifer, "The de finetti theorem for test spaces," e-print arXiv:quant-ph/0712.2265.

${ }^{12}$ C. D’Cruz, T. J. Osborne, and R. Schack, Phys. Rev. Lett. 98, 160406 (2007), e-print arXivquant-ph/0606139.

${ }^{13}$ R. König and G. Mitchison, "A most compendious and facile quantum de finetti theorem," J. Math. Phys. (to be published).

${ }^{14}$ R. L. Hudson and G. R. Moody, Z. Wahrscheinlichkeitstheor. Verwandte Geb. 33, 343 (1976).

${ }^{15}$ E. Størmer, J. Funct. Anal. 3, 48 (1969).

${ }^{16}$ C. M. Caves, C. A. Fuchs, and R. Schack, J. Math. Phys. 43, 4537 (2002), e-print arXivquant-ph/0104088.

${ }^{17}$ A. S. Holevo, Probabilistic and Statistical Aspects of Quantum Theory (North-Holland, New York, 1982).

${ }^{18}$ R. F. Werner and M. M. Wolf, Phys. Rev. Lett. 86, 3658 (2001).

${ }^{19}$ M. Fannes and C. Vandenplas, J. Phys. A 39, 13843 (2006), e-print arXivquant-ph/0605216.

${ }^{20}$ M. Ohya and D. Petz, Quantum Entropy and Its Use, Texts and Monographs in Physics (Springer, New York, 2004).

${ }^{21}$ M. M. Wolf, G. Giedke, and J. I. Cirac, Phys. Rev. Lett. 96, 080502 (2006).

${ }^{22}$ R. Renner, Nat. Phys. 3, 645 (2007), e-print arXiv:quant-ph/0703069.

${ }^{23}$ R. Garcia-Patron and N. J. Cerf, Phys. Rev. Lett. 97, 190503 (2006).
} 